\title{
Prevalencia de disfunción sexual en gestantes en control prenatal en una institución hospitalaria en Rionegro, Colombia, 2020-2021
}

\section{Prevalence of sexual dysfunction among pregnant women attending prenatal care at a hospital in Rionegro, Colombia, 2020-2021}

\section{Enrique Olivares-Noguera, $M D^{1}$; Rodrigo Montoya-Moreno, $M D^{I}$; Aníbal Arteaga Noriega, $\mathrm{MSc}^{2}$}

Recibido: 04 de agosto de 2021/Aceptado: 25 de noviembre de 2021

\section{RESUMEN}

Objetivo: describir la prevalencia de la disfunción sexual en un grupo de mujeres gestantes y hacer una exploración de posibles factores asociados a la disfunción sexual en estas mujeres.

Materiales y métodos: estudio descriptivo y trasversal en mujeres gestantes cuyas edades son de 15 años o más, con actividad sexual en la gestación, que asistieron al control prenatal en el Hospital San Juan de Dios de Rionegro entre los meses de enero y marzo del 2021. Se excluyeron pacientes con limitaciones o discapacidad cognitiva, o clasificadas con trastorno mental según la Organización Mundial de la Salud (OMS), gestantes con patologías crónicas, placentarias, ovulares, hemorrágicas e infecciosas, y las pacientes cuya gestación fue resultado de violencia sexual. Se aplicó el cuestionario Índice de Función Sexual Femenina (FSFI), se midieron variables sociodemográficas y de

* Correspondencia: Enrique Olivares Noguera, carrera 66 \# 35-04. Medellín, Antioquia. Correo electrónico: enriqueolivaresn@hotmail.com

1. Médico, residente de ginecología y obstetricia. Grupo de investigación en salud familiar y comunitaria, Corporación Universitaria Remington, Medellín (Colombia).

2. Enfermero, magíster en salud pública, estudiante de doctorado en epidemiología y bioestadística. Docente investigador del programa de enfermería, Facultad de Ciencias de la Salud, Corporación Universitaria Remington, Medellín (Colombia). salud sexual y reproductiva. Los resultados se expresan en frecuencias absolutas y relativas para las variables cualitativas y medianas, y rangos intercuartílicos para las variables cuantitativas.

Resultados: la mediana de edad fue de 27,5 años (RIC: 21,3-31,0); de edad gestacional 28,5 semanas (RIC: 21,3-34,8). Tras la aplicación del FSFI, 37 mujeres $(37,7 \%)$ tuvieron disfunción sexual (puntaje < 26,5). La mediana del puntaje de las participantes sin disfunción sexual fue de 29,4 (RIC 26,8-32), mientras que por el lado de las que tienen disfunción sexual fue de 22,3 (RIC 20-24). La media del puntaje para cada dominio fue: deseo: 3,6 (RIC: 3,0-4,2); excitación: 4,5 (RIC: 3,6-5,1); lubricación: 4,8 (RIC: 3,9-5,4); orgasmo: 4,4 (RIC: 3,6-5,2); dolor: 4,4 (RIC: 3,6-6,0); y satisfacción: 5,4 (RIC: 4,8-6,0). Fueron factores protectores no tener hijos ni cesáreas previas. La ausencia de compañero y menor edad estuvieron asociados a disfunción sexual.

Conclusión: la disfunción sexual se presenta en por lo menos un tercio de las gestantes, especialmente en el tercer trimestre. En la práctica clínica diaria se hace necesario abordar la disfunción sexual, ya que se puede contribuir desde el abordaje de la educación a 
un problema que puede afectar las relaciones de pareja y la calidad de vida. Es fundamental generar nuevas investigaciones que incluyan la evaluación de la efectividad y seguridad de estrategias educativas en este grupo poblacional con disfunción sexual.

Palabras clave: salud sexual, embarazo, sexo, disfunciones sexuales fisiológicas.

\section{ABSTRACT}

Objective: To describe the prevalence of sexual dysfunction in a group of pregnant women, and to explore potential factors associated with this condition in this population.

Material and methods: Descriptive cross-sectional study in pregnant women 15 years of age and older, sexually active during gestation, receiving prenatal care at the San Juan de Dios Hospital in Rionegro between January and March, 2021. The exclusion criteria were patients with disabilities or cognitive impairment, or classified as having a mental disorder according to the World Health Organization (WHO); pregnant women with chronic, placental, ovulation, hemorrhagic or infectious conditions; and patients whose pregnancy was the result of sexual assault. The Female Sexual Function Index (FSFI) questionnaire was applied, and sociodemographic and sexual and reproductive health variables were measured. Results are expressed as absolute and relative frequencies for qualitative variables, and as medians and interquartile ranges for quantitative variables.

Results: The mean age in years was 27.5 (IQR: 21.331.0) and the mean gestational age was 28.5 weeks (IQR: 21.3-34.8). After administering the FSFI, it was found that 37 women (37.7\%) had sexual dysfunction (score < 26.5). The median scores for the participants without sexual dysfunction and those with sexual dysfunction were 29.4 (IQR 26.8-32) and 22.3 (IQR 20-24), respectively. The mean scores for each domain were: desire 3.6 (IQR: 3.0-4.2); arousal 4.5 (IQR: 3.65.1); lubrication 4.8 (IQR: 3.9-5.4); orgasm 4.4 (IQR: 3.6-5.2); pain 4.4 (IQR: 3.6-6.0); and satisfaction 5.4 (IQR: 4.8-6.0). The total score $(\mathrm{p}<0.05)$ was better in older women and those with one or more children.
Conclusion: Sexual dysfunction occurs at least in onethird of pregnant women, especially during the third trimester. Sexual dysfunction should be considered in daily clinical practice, given that approaching the topic from an education perspective can help reduce a problem that affects the couple and quality of life. It is critical to undertake additional research that includes assessment of the effectiveness and safety of educational strategies in this population with sexual dysfunction.

Keywords: Sexual Health, pregnancy, sex, physiological sexual dysfunction.

\section{INTRODUCCIÓN}

De acuerdo con la investigadora Mariangela Carta Peña, la OMS define la salud sexual como "un estado de bienestar físico, mental y social en relación con la sexualidad, y no solamente la ausencia de enfermedad, disfunción o malestar. La adecuada función sexual requiere un enfoque positivo y respetuoso de la sexualidad y de las relaciones sexuales, así como la posibilidad de tener experiencias sexuales placenteras y seguras, libres de toda coacción, discriminación y violencia” (1). Para que todas las personas alcancen y mantengan una buena función y salud sexual, se deben respetar, proteger y satisfacer sus derechos sexuales (2).

La disfunción sexual ha sido definida como la disminución de los sentimientos de interés sexual, ausencia de deseo, pensamientos o fantasías sexuales ausentes, y una falta de deseo de respuesta (3). Afecta tanto a hombres como a mujeres, aunque ha sido más estudiada en hombres (4). En mujeres ha sido definida como la cadena de experiencias psiquiátricas, individuales y de pareja que se manifiesta como una disfunción en el deseo sexual, la excitación sexual, el orgasmo y el dolor durante el coito (5). Se describió que afectaba a cerca del 40\% de las mujeres de los Estados Unidos en 1992 (6). En Colombia, en el 2010 se describió que el 30\% de las mujeres entre 18 y 40 años tenían disfunción sexual (7). En cuanto a los factores que se han asociado a la disfunción sexual se encuentran factores biológicos, psicosociales, médicos, inter e intrapersonales (8). El diagnóstico de DS se hace por medio de escalas entre las cuales se encuentra el índice de disfunción sexual femenina (9), que ya ha sido validado en Colombia en 
lengua española (10). En cuanto al manejo, se basa en intervenciones psicológicas, farmacológicas, educativas y fisioterapia (11).

El periodo que abarca el embarazo resulta una de las etapas más importantes para toda mujer, en el que se producen cambios que repercutirán en su vida y, por lo tanto, en su sexualidad (2). En cuanto a los factores biológicos, se vuelve predictiva la disfuncionalidad sexual por razón de edad, aumento de peso (en relación con la autopercepción de atracción), aumento de secreción vaginal, paridad, la edad gestacional, antecedentes de aborto, infecciones y complicaciones durante el embarazo (12). Por otra parte, se deben tener en cuenta factores socioculturales que influyen en la sexualidad femenina en la gestación, tales como valores sociales, estado civil, además de las creencias y mitos frente a las prácticas sexuales. Los autores destacan las preocupaciones y temores respecto a afectar el desarrollo del embarazo, el nacimiento de un hijo con malformaciones, aborto espontáneo o hemorragias $(13,14)$. Por ejemplo, León y Arbués en su trabajo Características y evolución del patrón sexual de la mujer embarazada (15) afirman que una parte de los problemas de disfunción sexual percibidos durante el embarazo son atribuidos a la inadecuada educación y la falta de información al respecto, mitificándose la sexualidad como un factor que pone en riesgo el embarazo.

Con respecto al tiempo de embarazo se ha documentado que en todo el proceso de gestación se disminuye la satisfacción sexual y la frecuencia del coito. Por ejemplo, durante el primer trimestre de gestación en respuesta al influjo hormonal, factores somáticos y psicológicos, la excitabilidad y la satisfacción sexual varían considerablemente, por lo que cerca del $40 \%$ de mujeres sexualmente activas reportan disminución en el deseo sexual y, como consecuencia, alteración en su función sexual (15). Respecto al tercer trimestre, se ha descrito que la frecuencia de los contactos sexuales, el deseo sexual y la satisfacción disminuyen de manera importante y se ha puntualizado que la prevalencia de disfunciones sexuales en este trimestre puede llegar hasta el 71,94\% (4).

El diagnóstico y el tratamiento son similares a los que se utilizan en la mujer no gestante. Para las mujeres en estado de gestación, dependiendo de su condición clínica, se recomienda la práctica de relaciones sexuales con el fin de obtener beneficios psicológicos y una mejora del progreso del embarazo, pero si hay condiciones anormales se recomienda la restricción de dichas actividades con la finalidad de evitar complicaciones del embarazo (7).

Es poca la información sobre la prevalencia de la DSF en la gestación, especialmente en la región latinoamericana, como también respecto a los factores que incrementan la frecuencia de la misma en el embrazo, y que pueden variar de un país a otro por aspectos culturales(16). Por otra parte, hay limitaciones de estudios de prevalencia de las alteraciones en la función sexual de las mujeres en cuanto a la cobertura y el tipo de población incluida (17). Es importante que el obstetra explore esta disfunción en la gestación para poder ayudar a las mujeres que deseen continuar con su actividad sexual durante el embarazo. Por lo tanto, el objetivo de este estudio es describir la prevalencia de la disfunción sexual en un grupo de mujeres gestantes en Colombia y hacer una exploración de posibles factores asociados a la disfunción sexual en estas mujeres.

\section{MATERIALES Y MÉTODOS}

Diseño y población. Estudio transversal, descriptivo, en el que se incluyeron mujeres gestantes de 15 años o más, con actividad sexual en la gestación, que asistieron entre los meses de enero y marzo del 2021 al control prenatal en el Hospital San Juan de Dios de Rionegro, institución de mediana complejidad y referencia, que atiende a pacientes con aseguramiento subsidiado por el Estado en el Sistema de salud colombiano. Se excluyeron pacientes con limitaciones o discapacidad cognitiva, o clasificadas con trastorno mental según la OMS, gestantes con patologías crónicas previas al embarazo, o alteraciones placentarias, ovulares, hemorrágicas e infecciosas, y las pacientes cuya gestación fue resultado de violencia sexual. Se hizo muestreo por conveniencia, y no se realizó cálculo de tamaño de muestra.

Procedimiento. Un investigador (médico residente) abordó a las mujeres gestantes a la salida de su control prenatal, las invitó a participar y evaluó los criterios de inclusión. Si cumplían los criterios se les explicaba 
el objetivo de la investigación señalando los aspectos éticos y permitiendo la lectura del consentimiento informado para incluirla formalmente. Una vez firmado, la paciente fue llevada a un área privada, allí otro investigador aplicó el cuestionario para la toma de la información sobre las características sociodemográficas y obstétricas de las pacientes como también el instrumento de evaluación de la función sexual femenina. El investigador que aplicó el instrumento contó con el acompañamiento de una psicóloga especialista en el tema, con la finalidad de mejorar el abordaje de las pacientes que puedan presentar susceptibilidad emocional, y así poder ofrecerles una intervención primaria y tratar adecuadamente el caso. El instrumento para evaluar la función sexual fue el Índice de Función Sexual Femenina (FSFI, por sus siglas en inglés). Este instrumento se compone de 19 ítems evaluados en seis dominios principales: deseo (ítems 1 y 2), excitación (ítems 3 al 6), lubricación (ítems 7 al 10), orgasmo (ítems 11 al 13), satisfacción (ítems 14 al 16), y dolor (ítems 17 al 19). En esta investigación se utilizó la versión validada para Colombia (10) . La interpretación que se le da es que a mayor puntaje global mejor función sexual, y a menor puntaje mayor disfunción sexual. Se considera disfunción sexual si el puntaje total es menor a 26,5. Por otra parte, se puede analizar cada dominio por aparte y se toma la medida resumen como indicador de cuál de ellos está más afectado. Se buscó controlar el sesgo de información, generando un ambiente de confianza y privado para evitar alteraciones en el recuerdo y vergüenza por el tema a tratar. Se realizó verificación al 100\% de los registros para corregir datos faltantes y fuera de rango.

Variables medidas: sociodemográficas como edad, estado civil, nivel académico (secundaria o técnico/ universitario), ocupación, estrato socioeconómico (clasificación de ingresos utilizada en Colombia, a saber, estratos 1 y 2: ingresos bajos; 3 y 4 : ingresos medios; 5 y 6 ingresos altos); variables de salud sexual y reproductiva (paridad, número de partos vaginales, número de cesáreas, trimestre de embarazo); y antecedentes personales como consumo de fármacos, patológicos, quirúrgicos, e infecciosos. Se consideró disfunción sexual si el puntaje total fue menor a 25,6.
Se evaluaron los 6 dominios específicos por aparte, y se interpreta que a mayor puntaje es mejor el índice de sexualidad.

Análisis estadístico. Los datos fueron analizados en Jamovi 2.2.2; inicialmente se hizo análisis exploratorio de los datos para detectar aquellos con comportamiento atípico. La información de las variables cualitativas se resume por medio de frecuencias absolutas y relativas, y para las variables cuantitativas se presentaron las medidas de tendencia central y dispersión según su distribución mediante la prueba Shapiro Wilk (normal o no normal); se hace la comparación de las variables basales sociodemográficas entre el grupo de pacientes con y sin disfunción por medio de las pruebas de chi 2. Se utilizó una significancia de 0,05 . Se presenta la prevalencia de periodo de la DSF en el grupo estudiado y estratificada por trimestre de la gestación. Además, se presentan los puntajes globales en el grupo y en las mujeres que presentaron disfunción y aquellas que no la presentaron. Para el análisis exploratorio de los factores asociados a la disfunción sexual se categorizó en gestantes con disfunción (Puntaje $<26,5$ ), sin disfunción (puntaje $>$ o $=26,5$ ) y se evaluaron los factores que mostraron ser diferentes en la comparación de grupos por medio del Odds Ratio. Además, se evaluó la relación entre edad y puntaje por medio del coeficiente de correlación de sperman.

Aspectos éticos. La investigación cuenta con el aval del comité de ética investigación de la Corporación Universitaria Remington Acta 102020 y del hospital. Se solicitó la firma del consentimiento informado para incluir a las pacientes al estudio y se garantizó la privacidad y confidencialidad de la información.

\section{RESULTADOS}

De un total de 400 mujeres candidatas a ingresar, hubo contacto con 116, de las cuales se excluyeron 18 por no cumplir con los criterios de inclusión (3 por no tener relaciones sexuales durante el embarazo, 5 por causas infecciosas, 10 por patologías crónicas pre embarazo); en total se incluyeron 98 mujeres embarazadas, quienes aceptaron participar luego de la explicación y aclaración de las dudas. Todas ellas fueron tomadas en cuenta en el análisis. 
En cuanto a las características sociodemográficas de las mujeres entrevistadas se observó que la mediana de edad fue de 27,5 años (rango intercuartílico - RIQ 21,3 - 31). El 82,6\% de las pacientes tenían pareja. En cuanto a la formación, el $80 \%$ tuvo educación secundaria y el 20\% educación técnica/universitaria. El 83,7\% de las pacientes eran amas de casa, el 8,2\% eran empleadas y el otro 8,2\% eran independientes. El 76,5\% vivían en estrato socioeconómico 1 y 2, el 23,4\% vivían en estrato 3, 4 y 5 . La mediana de edad gestacional fue de 28,5 semanas (RIQ 21,3 - 34,8); el 51\% de las pacientes se encontraban en el tercer trimestre de la gestación y el $42,9 \%$ en el segundo trimestre. El 70,4\% de la población ya habían estado embarazadas. El 66,3\% tenían uno o más hijos vivos. El 56,1\% de las pacientes no había tenido un parto previo. El 26,5\% de las pacientes tenían antecedente de una o más cesáreas (Tabla 1). En cuanto a los antecedentes quirúrgicos, el 23,5\% tenían antecedente de cesárea, el 11,2\% de legrado obstétrico. El 19,4\% de las pacientes presentaron alguna infección durante el embarazo, el 12,2\% vaginosis, el 7,1\% infección de tracto urinario. En cuanto al consumo de medicamentos, solo el 11,2\% tenía algún tratamiento para manejo de patologías; el 5,1\% consumían levotiroxina, el 3,1\% ácido acetil salicílico. Al hacer la comparación de las variables basales sociodemográficas y clínicas por la presencia o no de DSF se encontró que los grupos difieren en cuanto al número de embarazos (p: 0,043), número de hijos (p: 0.004), cesáreas previas (p: 0,023), y estado civil (p: 0.049) (Tabla 1).

\begin{tabular}{|c|c|c|c|c|c|}
\hline \multicolumn{6}{|c|}{$\begin{array}{l}\text { Tabla 1. } \\
\begin{array}{c}\text { Características sociodemográficas y variables clínicas de mujeres con y sin disfunción sexual } \\
\text { en el embarazo, Rionegro, Colombia } 2021 .\end{array}\end{array}$} \\
\hline \multicolumn{3}{|c|}{ Con disfunción sexual } & \multicolumn{2}{|c|}{ Sin disfunción sexual } & \multirow[t]{2}{*}{ valor $\mathrm{p}$} \\
\hline VARIABLE & $\mathrm{n}=37$ & (\%) & $n=61$ & (\%) & \\
\hline Edad (Me-RIC) * & 25 & $(25-33)$ & 29 & $(20-30)$ & 0,017 \\
\hline Número de embarazos ** & & & & & 0,043 \\
\hline $0-1$ & 15 & 40,54 & 14 & 22,95 & \\
\hline 2 o más & 22 & 59,46 & 47 & 77,05 & \\
\hline Número hijos vivos ** & & & & & 0,004 \\
\hline 0 & 19 & 51,35 & 14 & 22,95 & \\
\hline 1 o más & 18 & 48,65 & 47 & 77,05 & \\
\hline Partos vaginales previos*** & & & & & 0,17 \\
\hline Ninguno & 13 & 35,14 & 30 & 49,18 & \\
\hline 1 o más & 24 & 64,86 & 31 & 50,82 & \\
\hline Sin cesáreas previas ** & 32 & 86,49 & 40 & 65,57 & 0,023 \\
\hline Estado civil: con pareja $* *$ & 27 & 72,97 & 54 & 88,52 & 0,049 \\
\hline Nivel académico ** & & & & & 0,92 \\
\hline Básica & 30 & 81,1 & 49 & 80 & \\
\hline Superior & 7 & 18,9 & 12 & 19 & \\
\hline Ocupación $* *$ & & & & & 0,98 \\
\hline Empleada & 6 & 15,51 & 10 & 16,39 & \\
\hline Ama de casa & 31 & 81,15 & 51 & 83,60 & \\
\hline Estrato socioeconómico ** & & & & & 0,049 \\
\hline $1--2$ & 28 & 75,68 & 47 & 77,04 & \\
\hline $3--4--5$ & 9 & 24,32 & 14 & 22,95 & \\
\hline Consumo medicamentos $* *$ & 7 & 18,9 & 4 & 6,56 & 0,060 \\
\hline Antecedentes patológicos ** & 11 & 29,73 & 12 & 19,67 & 0,25 \\
\hline Antecedentes quirúrgicos ** & 31 & 83,78 & 13 & 21,31 & 0,13 \\
\hline Infecciones en el embarazo $* *$ & 11 & 29,73 & 8 & 13,11 & 0,66 \\
\hline
\end{tabular}

*U de mann whitney.* *Chi cuadrado.

Fuente: elaboración propia. 
La prevalencia de DSF (puntaje menor a 26,5) fue del 37,7\% (37/98 mujeres). De ellas el 62,2\% estaban en el tercer trimestre, el 35,1\% estaban en el segundo y el 2,7\% estaban en el primero. La mediana del puntaje de las participantes sin disfunción sexual fue de 29,4 (RIC 26,8-32), mientras que por el lado de las que tienen disfunción sexual fue de 22,3 (RIC 20-24).

El puntaje para cada uno de los dominios del cuestionario fue el siguiente: la mediana de deseo fue de 3,6 (RIC 3,0-4,2), de excitación 4,5 (RIC 3,6-5,1), de lubricación 4,8 (RIC 3,9-5,4), de orgasmo 4,4 (RIC
3,6-5,2), de dolor 4,4 (RIC 3,6-6,0), y de satisfacción 5,4 (RIC 4,8-6,0).

Además, se identificaron posibles factores protectores contra la disfunción sexual, a saber, la ausencia de hijos previos OR: 0,28 (IC 0,11-0,67) y no haber tenido cesáreas previas OR: 0,29 (IC 0,12-0,87); como factor de riesgo se identificó el no tener pareja OR: 2,85 (IC 1,01-8,33). Respecto a la edad, encontramos que a mayor edad mejor puntuación ( $\mathrm{r}$ de spearman: 0,239; valor p: 0,018) (Tabla 2).

\begin{tabular}{|c|c|c|c|c|c|}
\hline \multirow[t]{2}{*}{ Variables } & \multirow[t]{2}{*}{ Categoría } & Disfunción sexual & $\begin{array}{c}\text { No disfunción } \\
\text { sexual }\end{array}$ & \multirow[t]{2}{*}{ OR } & \multirow[t]{2}{*}{ IC $95 \%$} \\
\hline & & $\mathbf{n}=37$ & $n=61$ & & \\
\hline \multirow{2}{*}{ Número de hijos } & 0 & 14 & 19 & \multirow{2}{*}{0,28} & \multirow{2}{*}{$0,11-0,67$} \\
\hline & $>1$ & 47 & 18 & & \\
\hline \multirow{2}{*}{ Cesáreas previas } & Sí & 32 & 40 & \multirow{2}{*}{0,29} & \multirow{2}{*}{$0,12-0,87$} \\
\hline & No & 5 & 21 & & \\
\hline \multirow{2}{*}{ Tiene pareja } & Sí & 27 & 54 & \multirow{2}{*}{2,85} & \multirow{2}{*}{$1,01-8,33$} \\
\hline & No & 10 & 7 & & \\
\hline Edad (rho) & & & & 0,018 & \\
\hline
\end{tabular}

Fuente: elaboración propia.

\section{DISCUSIÓN}

El estudio encontró una prevalencia de 37,7\% de disfunción sexual en las mujeres embarazadas que acudieron a control prenatal. La prevalencia fue mayor en el tercer trimestre y el dominio más afectado fue el deseo.

Respecto a la prevalencia de disfunción en el embrazo los resultados son similares a los informados por Prado y colaboradores en Brasil, quienes estudiaron en 177 gestantes la función sexual con el FSFI e informan una prevalencia de DSF del 40\% (18). En Chile, Osorio Cerda et al., informan en un centro de salud familiar prevalencia del 36\% en 50 gestantes, quienes se encontraban cerca de la mitad en el segundo trimestre de embarazo (19). Son inferiores a los publicados por Tosun y Gördele, quienes estudiaron en Turquía a 306 mujeres gestantes con embarazos de 28 semanas o más, con una prevalencia de DSF del 63\% (20); y a los informados por Daud et al. en 100 mujeres en Malasia con una prevalencia del 81\% de DSF en la gestación (21), y también a los resultados informados por JaramilloSaldarriaga et al. (22) en 256 mujeres gestantes, o en el posparto en Medellín Colombia con una prevalencia de DSF del 60\%.

En cuanto a la edad gestacional, el estudio encontró una proporción de disfunción sexual del 62\% en el tercer trimestre, resultado similar a lo encontrado por Ninivaggio en una muestra de 627 gestantes en Nuevo México, Estados Unidos, de las cuales 44\% eran latinas, y donde se encontró una prevalencia del 57\% de DSF en el tercer trimestre de la gestación en 
comparación al 36\% en el primer y segundo trimestre (23). Los resultados de este estudio no son consistentes con los informados en Egipto por Hanafy et al. En 300 gestantes, quienes encontraron que el 68\% de ellas tuvieron disfunción sexual en el primer trimestre de embarazo (24).

En cuanto a los puntajes por dominios parecidos a los resultados de este estudio se encuentra similitud en los informados por Espitia, quien también reporta que el dominio más afectado es el deseo con una puntuación por debajo de 3,8 en cada trimestre (25). Por otra parte, Hanfy informa que el deseo sexual se redujo en el primer trimestre $(3,5 \pm 1,2)$, la satisfacción sexual disminuyó en el primer trimestre $(4,2 \pm$ $1,1)$, en comparación con el segundo $(4,8 \pm 0,8)$ y el tercero $(4,6 \pm 1,0)(24)$. También son consistentes a los reportados por Muñoz y Camacho en 2014, en una revisión sistemática sobre disfunción sexual femenina en mujeres no embarazadas que reportaron que el dominio con mayor afectación fue el deseo (16).

Respecto a los factores de riesgo de DS en el embarazo, se encuentra poca información en la literatura internacional. Nuestros hallazgos son similares a los descritos en 118 mujeres gestantes por Karakas et al. (26), quienes asociaron la DS y la multiparidad; asimismo, informan la asociación de la DS con educación superior. Por otra parte, Soares et al. (27) en un estudio con 261 mujeres seleccionadas por conveniencia en Ceará, Brasil, asocian la DS con menor edad y un menor nivel de ingresos.

Debilidades del estudio: el muestreo por conveniencia limita la generalización de los resultados, y puede incrementar el riesgo de sesgos de selección. Como fortalezas tenemos el uso del IFSF validado en Colombia y hacer una aproximación más actual al problema en el país.

\section{CONCLUSIÓN}

La disfunción sexual se presenta en por lo menos un tercio de las gestantes, especialmente en el tercer trimestre en los dominios de excitación, dolor, orgasmo y satisfacción. En la práctica clínica diaria se hace necesario abordar esta problemática, ya que puede contribuir desde el abordaje o desde la educación a un problema que puede afectar las relaciones de pareja y la calidad de vida. Es fundamental generar nuevas investigaciones que incluyan la evaluación de la efectividad y seguridad de estrategias educativas en este grupo poblacional con disfunción sexual.

\section{REFERENCIAS}

1. Carta Peña M, González Blanco M. Función y patrón sexual: características y evolución durante el embarazo. Rev Obstet Ginecol Venez. 2016; 76(3):232-47.

2. Piñero Navero S, Alarcos Merinio G, Arenas Orta MT, Jiménez Iglesas V, Ortega Ballesteros EM, Ramiro Figueroa MJ. Características y modificaciones de la sexualidad durante el embarazo. NURE Inv. 2011;8(50).

3. Hatzimouratidis K, Hatzichristou D. Sexual dysfunctions: classifications and definitions. J Sex Med. 2007;4(1):241-250. https://doi.org/10.1111/j.17436109.2007.00409.x

4. Kadri N, Mchichi Alami KH, Mchakra Tahiri S. Sexual dysfunction in women: population based epidemiological study. Archives of Women's Mental Health. 2002;5(2):59-63. https://doi.org/10.1007/s00737002-0141-7

5. Wright JJ, O'Connor KM. Female Sexual Dysfunction. Medical Clinics of North America. 2015;99(3):60728. https://doi.org/10.1016/j.mcna.2015.01.011

6. Laumann EO, Paik A, Rosen RC. Sexual Dysfunction in the United States: Prevalence and Predictors. JAMA. 1999;281(6):537. https://doi.org/10.1001/ jama.281.6.537

7. Echeverry MC, Arango A, Castro B, Raigosa G. Study of the Prevalence of Female Sexual Dysfunction in Sexually Active Women 18 to 40 years of Age in Medellín, Colombia. The Journal of Sexual Medicine. 2010;7(8):2663-9. https://doi.org/10.1111/j.17436109.2009.01695.x

8. McCool-Myers M, Theurich M, Zuelke A, Knuettel $\mathrm{H}$, Apfelbacher C. Predictors of female sexual dysfunction: a systematic review and qualitative analysis through gender inequality paradigms. BMC Women's Health. 2018;18(1):108. https://doi.org/10.1186/ s12905-018-0602-4 
9. Rosen R, Brown C, Heiman J, Leiblum S, Meston C, Shabsigh R, et al. The Female Sexual Function Index (FSFI): A Multidimensional Self-Report Instrument for the Assessment of Female Sexual Function. Journal of Sex \& Marital Therapy. 2000;26(2):191-208. https://doi.org/10.1080/009262300278597

10. Vallejo-Medina P, Pérez-Durán C, Saavedra-Roa A. Translation, Adaptation, and Preliminary Validation of the Female Sexual Function Index into Spanish (Colombia). Arch Sex Behav. 2018;47(3):797-810. https://doi.org/10.1007/s10508-017-0976-7

11. American College of Obstetricians and Gynecologists. Female Sexual Dysfunction: ACOG Practice Bulletin Clinical Management Guidelines for ObstetricianGynecologists, Number 213. Obstetrics \& Gynecology. 2019;134(1):e1-18. https://doi.org/10.1097/ AOG.0000000000003324

12. Aslan G, Aslan D, Kızılyar A, İspahi Ç, Esen A. A prospective analysis of sexual functions during pregnancy. Int J Impot Res. 2005;17(2):154-7. https:// doi.org/10.1038/sj.ijir.3901288

13. Serati M, Salvatore S, Siesto G, Cattoni E, Zanirato M, Khullar V, et al. Female Sexual Function during Pregnancy and after Childbirth. J Sex Med. 2010;7(8):2782-90. https://doi.org/10.1111/j.17436109.2010.01893.x

14. Álvarez Leaño RK, Gutiérrez Fajardo Y, Velásquez Ríos DL. Vivencias de la sexualidad durante el embarazo [Tesis de pregrado]. Bogotá: Pontificia Universidad Javeriana; 2016. Disponible en: http://repository. javeriana.edu.co/handle/10554/20445

15. Rodríguez León M, Ramón Arbués E. Características y evolución del patrón sexual de la mujer embarazada. Enferm Glob. 2013; 12(32): 362-370. https://doi. org/10.6018/eglobal.12.4.181521

16. Muñoz D ADP, Camacho L PA. Prevalencia y factores de riesgo de la disfunción sexual femenina: revisión sistemática. Rev Chil Obstet Ginecol. 2016;81(3):168-80. https://doi.org/10.4067/S071775262016000300002

17. Aydin M, Cayonu N, Kadihasanoglu M, Irkilata L, Atilla MK, Kendirci M. Comparison of Sexual Functions in Pregnant and Non-Pregnant Women. Urol J. 2015;12(5):2339-44.
18. Prado DS, Lima RV, de Lima LM. Impacto da gestação na função sexual feminina. Rev Bras Ginecol Obstet. 2013;35(5):205-9. https://doi.org/10.1590/S010072032013000500003

19. Osorio Cerda L, Ortiz Lorca A, Muñoz Herrera P. Prevalencia de incontinencia urinaria y disfunción sexual en embarazadas atendidas en un Centro de Salud Familiar de Santiago de Chile. Rev Cubana Obstet Ginecol. 2018;44(1):1-11.

20. Tosun Güleroğlu F, Gördeles Beşer N. Evaluation of sexual functions of the pregnant women. J Sex Med. 2014;11(1):146-53. https://doi.org/10.1111/ jsm. 12347

21. Daud S, Zahid AZM, Mohamad M, Abdullah B, Mohamad NAN. Prevalence of sexual dysfunction in pregnancy. Arch Gynecol Obstet. 2019;300(5):12791285. https://doi.org/10.1007/s00404-019-05273-y

22. Jaramillo-Saldarriaga MI, Colonia-Toro A, Posada GA, Uribe MP. Factores asociados a disfunción sexual durante el embarazo: estudio multicéntrico en la ciudad de Medellín. Revista de la Facultad de Ciencias de la Salud Universidad del Cauca. 2021;23(1):15-22

23. Ninivaggio C, Rogers RG, Leeman L, Migliaccio L, Teaf D, Qualls C. Sexual function changes during pregnancy. Int Urogynecol J.2017;28(6):923-9. https:// doi.org/10.1007/s00192-016-3200-8

24. Hanafy S, Srour NE, Mostafa T. Female sexual dysfunction across the three pregnancy trimesters: an Egyptian study. Sex Health. 2014;11(3):240. https:// doi.org/10.1071/SH13153

25. Espitia de la Hoz FJ. Impacto del embarazo en la sexualidad de las gestantes del Eje Cafetero (COLOMBIA). Rev. chil. obstet. ginecol. 2020; 85(5): 537-548. https://doi.org/10.4067/S071775262020000500537

26. Karakas LA, Azemi A, Simsek SY, Akilli H, Esin S. Risk factors for sexual dysfunction in pregnant women during the COVID-19 pandemic. Int J Gynaecol Obstet. 2021;152(2):226-230. https://doi.org/10.1002/ ijgo. 13462

27. Soares PRAL, Calou CGP, Ribeiro SG, Aquino PS, Almeida PC, Pinheiro AKB. Sexuality and associated risk factors in pregnant women. Rev Bras Enferm. 2020;73 Suppl 4:e20180786. https://doi.org/10.1590/00347167-2018-0786 
Revista Colombiana de Obstetricia y Ginecología Vol. 72 No. 4 • 2021

\section{FINANCIACIÓN}

El presente estudio fue autofinanciado en su totalidad por los autores.

\section{CONTRIBUCIÓN DE LOS} AUTORES

Enrique Olivares-Noguera: elaboración y estructuración del documento desde su concepción y diseño hasta la adquisición de la información, revisión del contenido y aprobación de la versión enviada a proceso editorial; elaboración de tablas.

Rodrigo Montoya-Moreno: elaboración y estructuración del documento desde su concepción y diseño hasta la adquisición de la información.

Aníbal Arteaga-Noriega: elaboración y estructuración del documento desde su concepción y diseño hasta la adquisición de la información. 